\title{
Integrated microRNA and protein expression analysis reveals novel microRNA regulation of targets in fetal down syndrome
}

\author{
HUA LIN $^{1 *}$, WEIGUO SUI ${ }^{1 *}$, WUXIAN LI ${ }^{2 *}$, QIUPEI TAN ${ }^{1}$, JIEJING CHEN $^{1}$, XIUHUA LIN $^{2}$, \\ HUI GUO ${ }^{2}$, MINGLIN OU ${ }^{1}$, WEN XUE ${ }^{1}$, RUOHAN ZHANG $^{1}$ and YONG DAI ${ }^{2}$ \\ ${ }^{1}$ Nephrology Department of the 181st Hospital, Guangxi Key Laboratory of Metabolic Diseases Research, Guilin, \\ Guangxi 541002; ${ }^{2}$ The Second Clinical Medical College of Jinan University (Shenzhen People's Hospital), \\ Shenzhen, Guangdong 518020, P.R. China
}

Received August 23, 2015; Accepted September 9, 2016

DOI: $10.3892 / \mathrm{mmr} .2016 .5775$

\begin{abstract}
Down syndrome (DS) is caused by trisomy of human chromosome 21 and is associated with a number of deleterious phenotypes. To investigate the role of microRNA (miRNA) in the regulation of DS, high-throughput Illumina sequencing technology and isobaric tagging for relative and absolute protein quantification analysis were utilized for simultaneous expression profiling of miRNA and protein in fetuses with DS and normal fetuses. A total of 344 miRNAs were associated with DS. Gene Ontology and Kyoto Encyclopedia of Genes and Genomes pathway analyses were used to investigate the proteins found to be differentially expressed. Functionally important miRNAs were determined by identifying enriched or depleted targets in the transcript and the protein expression levels were consistent with miRNA regulation. The results indicated that GRB2, TMSB10, RUVBL2, the hsa-miR-329 and hsa-miR-27b, hsa-miR-27a targets, and MAPK1, PTPN11, ACTA2 and PTK2 or other differentially expressed proteins were connected with each other directly or indirectly. Integrative analysis of miRNAs and proteins provided an expansive view of the molecular signaling pathways in DS.
\end{abstract}

Correspondence to: Professor Yong Dai, The Second Clinical Medical College of Jinan University (Shenzhen People's Hospital), 1017 Dongmen North Road, Shenzhen, Guangdong 518020, P.R. China

E-mail: daiyong2222@gmail.com

Abbreviations: DS, Down syndrome; miRNA, microRNA; PAGE, polyacrylamide gel electrophoresis; RT-qPCR, reverse transcription-quantitative polymerase chain reaction; GO, gene ontology; ITRAQ, isobaric tagging for relative and absolute protein quantification

*Contributed equally

Key words: Down syndrome, microRNAs, high-throughput nucleotide sequencing, proteomics, bioinformatics

\section{Introduction}

Genomic copy variations, including copy number variations and chromosome aneuploidies, offer biological diversity and lead to genetic disorders. Down syndrome (DS) is caused by trisomy of human chromosome 21 (chr21) and is associated with numerous deleterious phenotypes, including cognitive impairment, childhood leukemia and immune defects (1). It occurs in $~ 1 / 700$ newborns worldwide (2). As the genetic basis for DS is known as an extra copy of chr21, several studies have focused on genes located on chr21. A number of genes located on chr21 are expressed at high levels in individuals with DS (3), however, several genes on other chromosomes are also disordered (4). Understanding the mechanism underlying how the extra chr21 causes various disease phenotypes can lead to improved management and, in the long term, treatment outcomes for individuals with DS. It is important to identify a safe and effective method to identify novel potential biomarkers.

MicroRNAs (miRNAs) are a class of small RNAs of $\sim 22 \mathrm{nt}$, which are important in a number of key biological processes and several human diseases at the post-transcriptional level of gene expression (5). Previous studies have shown that miRNAs are important in the normal regulation of gene expression during cell proliferation and development (6). Identification of the differential expression of genome-wide known and novel miRNAs can facilitate in uncovering the molecular regulatory mechanisms underlying the progression of the complex and variable phenotype of DS. A previous finding suggested that miR-1246 may serve as a likely link between the p53 family and Down syndrome (7). Previous studies have focused predominantly on the Hsa21-derived miRNAs and have been performed on the tissues of humans with DS, whereas few studies have focused on the expression profile of miRNAs isolated from human blood samples (8-10), and investigation of novel methods in this area is warranted.

Elkan-Miller et al (11) used a novel method for the identification of functionally important miRNA-target interactions, integrating transcriptome, proteome and miRNA profiles, and advanced in silico analysis using the Functional Assignment of miRNA via Enrichment algorithm. These miRNAs were determined by identifying depleted or enriched targets in the 
protein and transcript datasets, with an expression consistent with the accepted model of miRNA regulation. To obtain an unbiased and complete view of the small RNA transcriptome and further investigate the role of miRNAs in early embryonic development of the DS fetus, the present study investigated the regulation of protein and miRNA expression as an initial step towards a better understanding of the regulation of gene expression in DS. The aim was to provide an expansive view of DS from the integrated bioinfomatics analysis of proteomics and miRNA data sets.

\section{Materials and methods}

Patients and controls. A total of six DS and six matched control fetal cord blood samples (18-22 weeks of gestation) were obtained from the Shenzhen People's Hospital (Shenzhen, China). The diagnosis of DS was confirmed through chromosome examination. The six DS and six control cord blood samples were combined to form pooled DS and control cord blood samples, respectively, for small RNA library construction and Illumina sequencing. The cord blood samples were obtained by puncture extraction with the assistance of a color Doppler ultrasound as the prenatal women were undergoing prenatal diagnosis. The characteristics of each case are provided in the Table I. The present study was approved by the Ethics Committee of Shenzhen People's Hospital. The CBMCs were separated using Ficoll-Paque (Sigma-Aldrich; Thermo Fisher Scientific, Inc., Waltham, MA, USA) density gradient centrifugation according to the manufacturer's protocol. Briefly, $2 \mathrm{ml}$ of blood was layered on $3 \mathrm{ml}$ of Ficoll-Paque and centrifuged for $25 \mathrm{~min}$ at $1,000 \mathrm{x} g$ at room temperature. Mononuclear cells were aspirated with a pipette, washed twice in phosphate-buffered saline by centrifugation for $10 \mathrm{~min}$ at $700 \mathrm{x} g$ at room temperature and dissolved in $1 \mathrm{ml} \mathrm{TRIzol}{ }^{\circledR}$ reagent (Invitrogen; Thermo Fisher Scientific, Inc.). These samples were stored at $-80^{\circ} \mathrm{C}$ until further use (12).

The total plasma protein was extracted, and the concentration was measured using a BCA protein kit (Pierce Biotechnology, Rockford, IL, USA). In the present study, prior to proteomic analysis, $40 \mu \mathrm{g}$ of protein from each sample in each group was pooled.

Written informed consent was obtained from all guardians or subjects involved. The use of material for experiments was approved by the Ethics Committee of 181 Hospital (Guilin, China). The study was performed in accordance with the Helsinki Declaration on ethical principles for medical research involving human subjects.

Deep sequencing. Total RNA isolation from the CBMCs was performed using TRIzol reagent (Invitrogen; Thermo Fisher Scientific, Inc.) according to the manufacturer's protocol. Small RNA library preparation and sequencing were performed using Illumina sequencing technology (BGI, Shenzhen, China). In brief, the small RNA was isolated by separating $10 \mu \mathrm{g}$ of the total RNA using denaturing polyacrylamide gel electrophoresis (PAGE) and excising the region of the gel corresponding to 15-30 nt, based on standard oligonucleotide markers. Small RNAs were then reverse transcribed to cDNA using miRNA-specific stem-loop-like reverse transcription primers and amplified by the ABI PRISM 7500 Sequence Detection System (Applied
Biosystems; Thermo Fisher Scientific, Inc). Finally, the amplified cDNAs were purified on a $6 \%$ Tris-Borate-EDTA PAGE and were sequenced on the Illumina Hi-seq 2000 system (Illumina, Inc., San Diego, CA, USA). Two small RNA sequencing data sets comprising the DS and control CBMCs were obtained from Illumina fast track sequencing services. The frequencies of each small RNA sequence reads were calculated as sequence tags, and only sequences of 18-30 nt were retained for further analysis. All unique sequence reads, which passed above the filters were mapped onto the reference human genome using the SOAP (version 2.0) program (www.bioconductor. org/packages/2.4/bioc/html/KEGGSOAP.html) with at most two mismatches (13). The differential expression of miRNAs was calculated by relative expression analysis between the DS and control.

Reverse transcription-quantitative polymerase chain reaction (RT-qPCR) analysis. RT-qPCR was performed as described previously with a minor modification (14). In brief, total RNA was isolated from the CBMCs using TRIzol reagent (Invitrogen; Thermo Fisher Scientific, Inc.) according to the manufacturer's protocol. The RNA was then reverse transcribed into cDNA using miRNA-specific stem-loop like RT primer (GenePharma, Shanghai, China). PCR was performed using an Applied Biosystems 7500 real-time PCR machine (Applied Biosystems; Thermo Fisher Scientific, Inc.). The PCR reaction was conducted at $95^{\circ} \mathrm{C}$ for $5 \mathrm{~min}$, followed incubation at $95^{\circ} \mathrm{C}$ for $15 \mathrm{sec}, 65^{\circ} \mathrm{C}$ for $15 \mathrm{sec}$ and $72^{\circ} \mathrm{C}$ for $32 \mathrm{sec}$ for 40 cycles using SYBR Green PCR Master Mix (Toyobo Co., Ltd., Osaka, Japan). Each PCR was repeated at least three times. The relative expression level of each miRNA was normalized against the level of RNU6B. Fold-changes were calculated according to the $2^{-\Delta \Delta \mathrm{Cq}}$ method (14).

Isobaric tagging for relative and absolute protein quantification (iTRAQ) strong cation exchange (SCX)-tandem mass spectrometry (MS/MS) analysis. The total protein of each corresponding group was blocked, digested and labeled using the iTRAQ protocol (Applied Biosystems; Thermo Fisher Scientific, Inc.). The labeled digests were combined into each sample mixture. Multidimensional liquid chromatography was used to separate the tryptic peptides prior to MS. The combined samples were separated into 10 SCX fractions using a $300 \AA$, $35 \times 0.3 \mathrm{~mm}, 3.5-\mu \mathrm{m}$ particle size column (Zorbax Bio-SCX; Agilent Technologies, Inc., Santa Clara, CA, USA) with a potassium formate gradient in $25 \%$ acetonitrile. The peptides in these fractions were separated on Tempo ${ }^{\mathrm{TM}} \mathrm{LC}$ nanoflow and MALDI spotting systems (Applied Biosystems; Thermo Fisher Scientific, Inc.) equipped with a reversed-phase Magic C18AQ column (Phenomenex, Inc., Torrance, CA, USA). Each chromatography run yielded \%380 MALDI spots on a stainless steel MALDI target plate. MS data acquisition was calculated using an Applied Biosystems 4800 Plus MALDI TOF/TOF analyzer (Applied Biosystems; Thermo Fisher Scientific, Inc.). Only a signal to noise ratio $\geq 40$ was selected for $\mathrm{MS} / \mathrm{MS}$. Mass spectra from 500 laser shots were obtained for each spot. The combined MS/MS data from 10 SCX fractions was used for a Paragon algorithm search engine and human V3.62 (European Bioinformatics Institute; www.ebi.ac.uk) (15). 
Table I. Characteristics of each case.

\begin{tabular}{lccc}
\hline Mother ID & Age (years) & $\begin{array}{c}\text { Gestational } \\
\text { age (weeks) }\end{array}$ & $\begin{array}{c}\text { Karyotype } \\
\text { result }^{\mathrm{a}}\end{array}$ \\
\hline Patient 1 & 44 & 20 & $47, \mathrm{XX},+21$ \\
Patient 2 & 36 & 20 & $47, \mathrm{XX},+21$ \\
Patient 3 & 30 & 21 & $47, \mathrm{XY},+21$ \\
Patient 4 & 34 & 20 & $47, \mathrm{XX},+21$ \\
Patient 5 & 33 & 22 & $47, \mathrm{XY},+21$ \\
Patient 6 & 32 & 19 & $47, \mathrm{XX},+21$ \\
Control 1 & 37 & 21 & $46, \mathrm{XX}$ \\
Control 2 & 36 & 20 & $46, \mathrm{XY}$ \\
Control 3 & 30 & 20 & $46, \mathrm{XX}$ \\
Control 4 & 34 & 18 & $46, \mathrm{XY}$ \\
Control 5 & 33 & 22 & $46, \mathrm{XX}$ \\
Control 6 & 32 & 21 & $46, \mathrm{XX}$ \\
\hline
\end{tabular}

${ }^{a}$ The karyotype result indicates the total number of chromosomes, the sex chromosomes and the number of the extra chromosome.

\section{Bioinformatics analysis}

miRNA expression profile and targetgene prediction. Three software programs were used to predict target genes: miRanda v5 (www.ebi.ac.uk/enright-srv/microcosm/htdocs/targets/v5/), TargetScan 5.1 (www.targetscan.org) and PicTar 2005 (pictar. mdc-berlin.de/cgi-bin/PicTar_vertebrate.cgi) (16).

The results were predicted by the three software programs at the same time and was considered reliable. The differentially expressed proteins were identified using iTRAQ analysis. Standard human gene symbols of these proteins were used to search the list of miRNA-targeted genes. Cytoscape software was then used to obtain the miRNA and target gene regulation network.

Protein expression profile and gene interaction regulatory network. The differentially expressed proteins were analyzed using the Mammalian Protein-Protein Interaction (MIPS) database (mips.helmholtz-muenchen.de/proj/ppi/), Kyoto Encyclopedia of Genes and Genomes (KEGG) SOAP and co-citation calculation in PubMed (ncbi.nlm.nih.gov/pubmed). A network was constructed by integrating the results of the differentially expressed proteins analyzed using the MIPS database. (17).

Gene ontology (GO) and KEGG pathway analysis. To further understand the functions of the identified proteins, the present study used the online GO tool, Web Gene Ontology Annotation Plot (WEGO; http://wego.genomics.org.cn/). GO and KEGG pathway mapping of the targeted genes were performed using the web-accessible Database for Annotation, Visualization and Integrated Discovery (DAVID) annotation system (david. ncifcrf.gov).

\section{Results}

miRNA expression profile. To investigate the expression profile of genome-wide miRNA in the umbilical cord blood (UCB),
Table II. Interaction count analysis of the target gene network.

\begin{tabular}{|c|c|}
\hline Target gene & $\begin{array}{l}\text { Interaction } \\
\text { count }\end{array}$ \\
\hline PSMA4 & 5 \\
\hline PTK2 & 10 \\
\hline PSMA6 & 6 \\
\hline COL1A2 & 7 \\
\hline C4B & 6 \\
\hline YWHAG & 6 \\
\hline EIF3I & 6 \\
\hline MAPK1 & 11 \\
\hline SF3B1 & 5 \\
\hline CAV1 & 7 \\
\hline SERPINH1 & 5 \\
\hline SCARB2 & 5 \\
\hline TMSB10 & 14 \\
\hline RPL30 & 5 \\
\hline RUVBL2 & 8 \\
\hline PTPN11 & 8 \\
\hline GRB2 & 13 \\
\hline PSMA3 & 6 \\
\hline ACTA2 & 15 \\
\hline LAMB2 & 6 \\
\hline ALB & 18 \\
\hline PLG & 16 \\
\hline UBE2I & 5 \\
\hline FN1 & 19 \\
\hline SUMO2 & 7 \\
\hline MARCKS & 5 \\
\hline EIF3G & 6 \\
\hline RPS14 & 6 \\
\hline RUVBL1 & 8 \\
\hline CRK & 6 \\
\hline PSMD1 & 7 \\
\hline PSMD13 & 6 \\
\hline SNRPD2 & 6 \\
\hline
\end{tabular}

Genes with an interaction count $\geq 5$ are included. Interaction count indicates the number of interactions of a gene with other genes. If the number is higher, the target gene is considered of higher significance in the network.

the present study used Illumina sequencing technology to sequence the small RNA libraries of the DS group and normal group. A total of 344 miRNAs were detected as being differentially expressed, if which 46 miRNAs were upregulated and 298 miRNAs were downregulated in DS, compared with the normal control group. To validate the results of the Illumina sequencing, RT-qPCR assays were performed with specific stem-loop RT primers to examine the expression levels of the Hsa21-derived mature miRNAs and randomly selected significantly differentially expressed miRNAs, including four downregulated miRNAs (hsa-miR-16, miR-126, miR-21 and miR-223) and two upregulated miRNAs (hsa-miR-196b and 


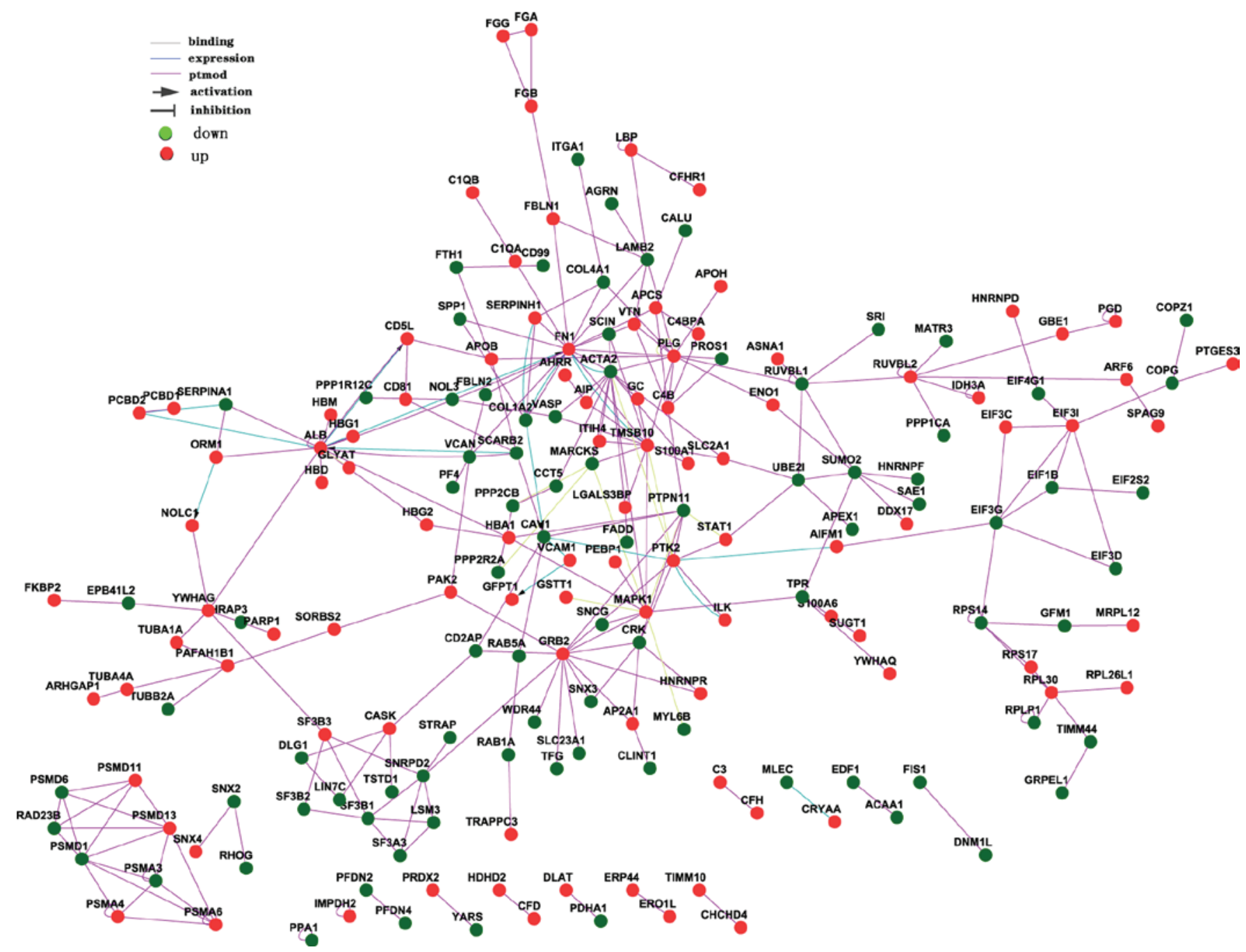

Figure 1. Gene interaction regulatory network in Down syndrome. Green and red circles indicate downregulated and upregulated differentially expressed proteins (target gene), respectively. Gray lines indicate binding activity; blue lines indicate expression regulatory activity; purple lines indicate post-transcription modification activity.

miR-92b*). The results of the RT-qPCR analysis indicated similar expression levels of the miRNAs to the deep sequencing.

Protein expression profile and gene interaction regulation network. To investigate the expression profile of proteins in the plasma of the UCB, the present study used iTRAQ technology. Relative quantification of proteins was based on the ratio of peak areas from the MS/MS spectra. Compared with the control group, 505 differentially expressed proteins were identified, including 250 downregulated and 255 upregulated proteins, with tryptic peptides differing 1.5 -fold $(\mathrm{P}<0.05)$ in the DS group. The differentially expressed proteins were analyzed using the MIPS database, KEGGSOAP and co-citation calculation in PubMed. The interaction regulation network was constructed by integrating the results of the these three types of data following comprehensive considerations (Fig. 1). The network interaction count is listed in Table II, which indicates the interaction count of a gene with other genes.

Differentially expressed gene and differentially expressed miRNA association analysis. From the DS and normal control CBMCs, the present study identified 344 miRNAs with significantly differing levels of expression. These miRNAs targets were examined using the three software programs mentioned above. The predicted targets of 58 miRNAs, including hsa-miR-27b, hsa-miR-329 and hsa-miR-27a, with the highest total context score are listed in Table III. The predicted targets were only found in the list of differentially expressed proteins using iTRAQ analysis. Cytoscape software was then used to obtain the miRNA and target gene association regulation network (Fig. 2).

GO and KEGG pathway analysis. With the aim of elucidating the specific function of miRNAs significant to the embryonic development of DS, the present study annotated the predicated targets with GO schemes using the DAVID gene annotation tool. The genes of proteins potentially regulated by differentially expressed miRNAs produced a total of $37 \mathrm{GO}$ terms in DS (Table IV), including 11 in biological process, 13 in cellular component and 13 in molecular function. By examining the GO 'biological process' classifications, the significant GO terms $(\mathrm{P}<0.01)$ were genes involved in GO:0006519 cellular amino acid and derivative metabolic process (24), GO:0006810 transport (85) and GO:0016043 cellular component organization 
Table III. Predicted miRNA targets from three commonly used software programs in Down syndrome.

\begin{tabular}{|c|c|}
\hline miRNA & Target gene \\
\hline \multicolumn{2}{|l|}{ Downregulated } \\
\hline hsa-miR-142-3p & $\begin{array}{l}\text { RAB2A, LLGL2 } \\
\text { TFG, COPG }\end{array}$ \\
\hline hsa-miR-197 & SSR3, OTUB1 \\
\hline hsa-miR-223 & PARP1, POLDIP2 \\
\hline hsa-miR-139-5p & FGA \\
\hline hsa-miR-150 & BASP1 \\
\hline hsa-miR-192 & RAB2A \\
\hline hsa-miR-16 & $\begin{array}{l}\text { YWHAQ, TUBA1A } \\
\text { SNCG, VAMP8 } \\
\text { AP2A1, STXBP3 }\end{array}$ \\
\hline hsa-miR-874 & PPP1CA \\
\hline hsa-miR-590-3p & RAD23B, PPA1 \\
\hline hsa-miR-485-5p & MGST3 \\
\hline hsa-miR-132 & $\begin{array}{l}\text { PPP2CB, LRRFIP1 } \\
\text { SLC2A1, CRK, FKBP2 }\end{array}$ \\
\hline hsa-miR-431 & RBPMS \\
\hline hsa-miR-411 & SF3B3 \\
\hline hsa-miR-24 & MPI, CRAT \\
\hline hsa-miR-543 & CASK \\
\hline hsa-miR-30a & UBE2I, AP2A1 \\
\hline hsa-miR-30e & UBE2I, AP2A1 \\
\hline hsa-miR-329 & GRB2 \\
\hline hsa-miR-25 & COL1A2, PPP1R12C \\
\hline hsa-miR-377 & RBPMS, OGDHL \\
\hline hsa-miR-374b & CALU \\
\hline \multirow[t]{2}{*}{ hsa-miR-107 } & VAMP8, SNCG \\
\hline & SNX3, UMOD \\
\hline hsa-miR-26a & MICAL3, COL1A2 \\
\hline hsa-miR-379 & YARS \\
\hline \multirow[t]{3}{*}{ hsa-miR-29c } & DNAJB11, ARF5 \\
\hline & COL4A1, COL1A2 \\
\hline & COL1A2, HMGN3, TUBB2A \\
\hline hsa-miR-376a & SUGT1, DLAT \\
\hline \multicolumn{2}{|l|}{ Upregulated } \\
\hline hsa-miR-206 & $\begin{array}{l}\text { DDX5, HMGN1, SNX2, RRBP1 } \\
\text { DDX17, TRAPPC3, PGD }\end{array}$ \\
\hline hsa-miR-196b & CASK, COL1A2 \\
\hline hsa-miR-183 & PPP2CB \\
\hline hsa-miR-424 & $\begin{array}{l}\text { TUBA1A, SNCG, VAMP8 } \\
\text { AP2A1, CALU, STXBP3 }\end{array}$ \\
\hline hsa-miR-31 & PPP2R2A \\
\hline hsa-miR-324-5p & RAN \\
\hline hsa-miR-224 & DNAJC8, ZNF207 \\
\hline hsa-miR-28-5p & PSAP, OTUB1 \\
\hline hsa-miR-23b & CFDP1, ENTPD5, PRDX3 \\
\hline hsa-miR-27b & $\begin{array}{l}\text { TMSB10, RUVBL2, FBLN2 } \\
\text { XPO1, ACTA2 }\end{array}$ \\
\hline hsa-miR-494 & PFDN4, F11R, ZNF207 \\
\hline hsa-miR-145 & C6ORF115, ARPC5, AARS \\
\hline
\end{tabular}

Table III. Continued.

\begin{tabular}{|c|c|}
\hline miRNA & Target gene \\
\hline hsa-miR-363 & VPS4B, COL1A2, PPP1R12C \\
\hline hsa-miR-27a & $\begin{array}{l}\text { TMSB10, RUVBL2 } \\
\text { FBLN2, ACTA2 }\end{array}$ \\
\hline hsa-miR-101 & RAB5A, ZNF207, FGA \\
\hline hsa-miR-22 & ENO1 \\
\hline hsa-miR-30d & UBE2I, AP2A1 \\
\hline hsa-miR-410 & TRAPPC3, CASK \\
\hline hsa-miR-495 & RAN, SEPT7 \\
\hline hsa-miR-499-5p & ERO1L, MARCKS, EPB41L2 \\
\hline hsa-miR-186 & EFEMP1, DNAJC8, TPR \\
\hline hsa-miR-539 & DDX5 \\
\hline hsa-miR-376b & SUGT1, DLAT \\
\hline hsa-miR-23a & CFDP1, ENTPD5 \\
\hline hsa-miR-195 & $\begin{array}{l}\text { YWHAQ, TUBA1A, SNCG } \\
\text { VAMP8, AP2A1, STXBP3 }\end{array}$ \\
\hline hsa-miR-30c & UBE2I, AP2A1 \\
\hline hsa-miR-221 & ARF4 \\
\hline hsa-miR-29a & $\begin{array}{l}\text { DNAJB11, ARF5, COL4A1 } \\
\text { HMGN3, TUBB2A } \\
\text { XPNPEP1 }\end{array}$ \\
\hline hsa-miR-30b & UBE2I, AP2A1 \\
\hline hsa-miR-29b & $\begin{array}{l}\text { HMGN3, DNAJB11, TUBB2A } \\
\text { ARF5, COL1A2 }\end{array}$ \\
\hline hsa-miR-92b & RRBP1, PPP1R12C \\
\hline hsa-miR-182 & ARF4 \\
\hline
\end{tabular}

Results are the predictions of three software programs (PicTar, miRanda and TargetScan) at the same time, and was considered reliable. $\mathrm{miR} / \mathrm{miRNA}$, microRNA.

(84). The significant cellular component GO terms were genes involved in GO:0005829 cytosol (71), GO:0005739 mitochondrion (46), GO:0005768 endosome (15) and GO:0005794 Golgi apparatus (26). Molecular function ontology showed GO:0005515 protein binding (137), GO:0005198 structural molecule activity (23) and GO:0019825 oxygen binding (4). The GO terms indicated that GRB2, TMSB10 and RUVBL2, the hsa-miR-329 and hsa-miR-27b, hsa-miR-27a targets, and the differentially expressed proteins were connected with each other either directly or indirectly. There was a direct association between GRB2 and MAPK1, PTK2 and PTPN11. There was also a direct association between TMSB10, RUVBL2 and ACTA2. These results suggested that a set of abundant and significantly differentially expressed miRNAs may promote the progression of cognitive impairment in patients with DS by regulating genes in the pathway of nervous system development.

In addition, the present study obtained 28 KEGG pathways of the differentially expressed proteins in DS (Table V), including 'Focal adhesion' (Fig. 3), which was significantly enriched $(\mathrm{P}<0.05)$. The potential network of 'Focal adhesion' indicated that GRB2, hsa-miR-329 targets and the differentially expressed proteins were connected with each other, 


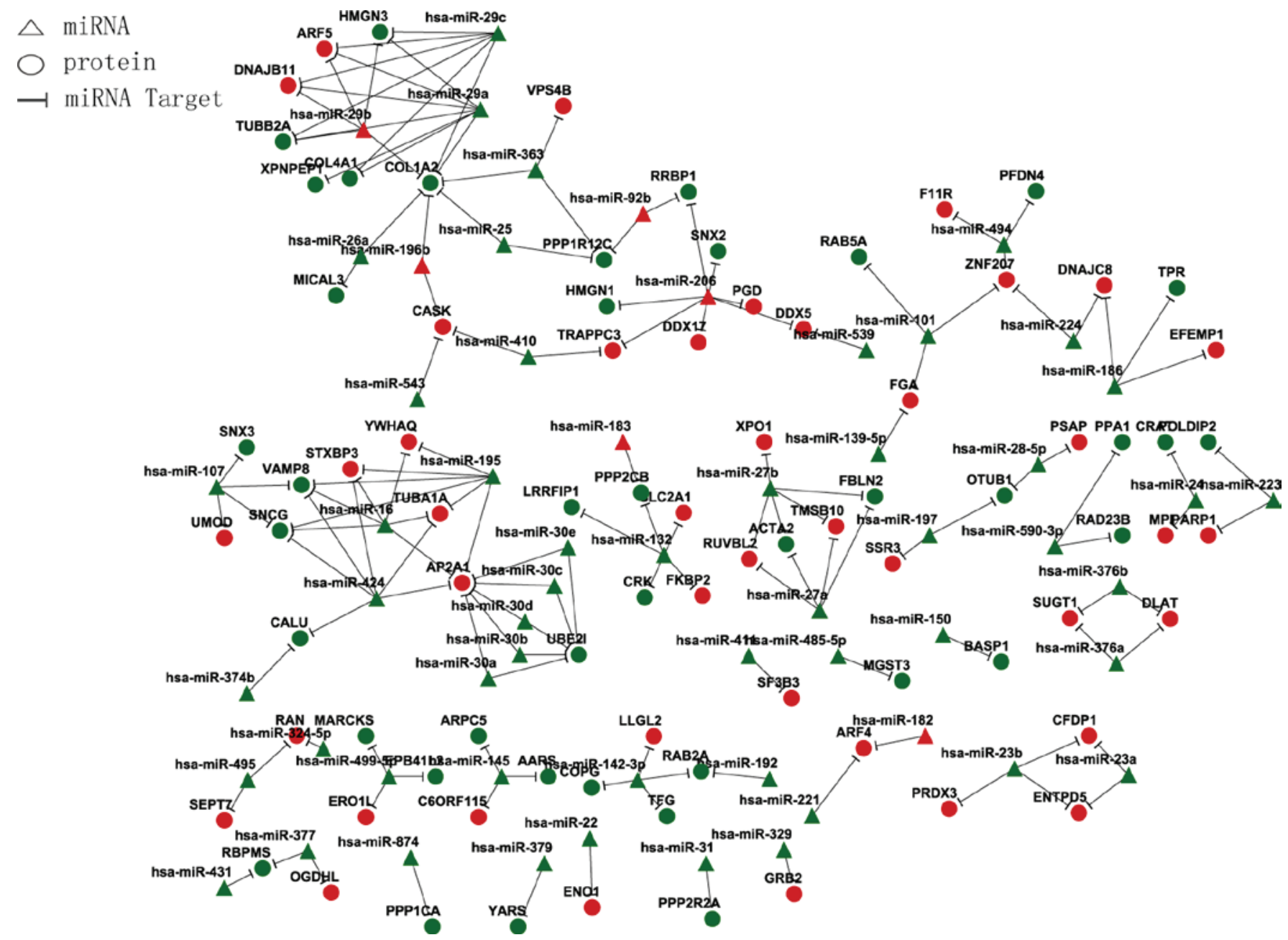

Figure 2. miRNA and target gene association regulatory network in Down syndrome. The miRNA target gene and proteins were considered reliable following prediction by three software programs at the same time. The network was limited to targets predicted by all three software programs. Triangles represent miRNAs, circles represent proteins. Green and red indicate downregulated and upregulated differentially expressed proteins or miRNAs, respectively. miRNA, microRNA.

either directly or indirectly; and there was a direct association of co-citation between GRB2 and MAPK1 involved in the MAPK signaling pathway. This suggested that the significantly differentially expressed proteins may promote the progression of cognitive impairment in patients with DS.

\section{Discussion}

The miRNA-guided regulation of gene expression may involve hundreds of miRNAs and their targets in animals. Genetic studies have successfully identified termed genetic switches of certain miRNA activities, which have intrinsic phenotypic consequences (18). miRNAs are crucial in post-transcription regulation, and the extra hsa21 in DS leads to the disordered expression of genes. The present study aimed to identify the protein and miRNA profiles, and reveal potential miRNA-targets in fetal DS using a combinatorial and novel approach involving iTRAQ quantitative proteomics, deep sequencing and bioinformatic analysis. A total of 58 known miRNAs were detected to have significantly different expression, which may be involved in the variable phenotypes of DS. Their predicted targets were found in the list of the differentially expressed proteins using iTRAQ analysis. This may indicate a general connection between miRNA regulation of their coding genes and functional complexity of proteins. The present study also integrated miRNA and protein datasets and identified the three most differentially expressed miRNAs, seven differentially expressed proteins and one KEGG pathway in the DS fetus group. Functional analysis of miRNAs in DS is required.

miRNAs are involved in gene regulation, and have been recognized as predictive tools and important intervention targets for several diseases due to the convenience and stability of miRNA detection (19). In the present study, the challenge was to map miRNAs to specific gene targets and the molecular networks they regulate. Studies have provided insights into miRNA-mediated gene regulation in Ts65Dn mice, and the potential contribution to impaired hippocampal synaptic plasticity and neurogenesis, and the hemopoietic abnormalities observed in DS $(20,21)$. The present study found evidence for the functional importance of several previously unknown miRNAs in DS. Specific miRNA expression profiles may point to the particular role of the miRNA in DS. miR-329 can inhibit cell proliferation in human glioma cells through regulating E2F1 (22). miRNA target genes can regulate cell development and differentiation, cell cycle, and apoptosis and miRNAs, 
Table IV. Differentially expressed proteins annotation terms of the GO molecular function, cellular component and biological process categories in Down syndrome.

\begin{tabular}{|c|c|c|c|c|}
\hline Term & P-value & $\begin{array}{c}\text { Upregulated } \\
\text { (downregulated) } \\
\text { genes }(\mathrm{n})\end{array}$ & $\begin{array}{l}\text { Significantly } \\
\text { upregulated } \\
\text { genes }\end{array}$ & $\begin{array}{c}\text { Significantly } \\
\text { downregulated } \\
\text { genes }\end{array}$ \\
\hline \multicolumn{5}{|l|}{ Biological process } \\
\hline $\begin{array}{l}\text { Cellular amino acid and } \\
\text { derivative metabolic process }\end{array}$ & $2.08 \mathrm{E}-06^{\mathrm{a}}$ & $11(13)$ & & \\
\hline Transport & $3.20 \mathrm{E}-06^{\mathrm{a}}$ & $49(36)$ & MAPK1, GRB2 & \\
\hline $\begin{array}{l}\text { Cellular component } \\
\text { organization }\end{array}$ & $0.001292^{\mathrm{a}}$ & $46(38)$ & $\begin{array}{l}\text { TMSB10, RUVBL2, MAPK1, } \\
\text { GRB2 }\end{array}$ & PTPN11 \\
\hline Translation & 0.069213 & $6(6)$ & MAPK1 & \\
\hline Protein modification process & 0.216467 & $25(12)$ & RUVBL2, MAPK1 & PTPN11 \\
\hline $\begin{array}{l}\text { Multicellular organismal } \\
\text { development }\end{array}$ & 0.288361 & $33(32)$ & MAPK1, GRB2 & PTPN11 \\
\hline Carbohydrate metabolic process & 0.415425 & $9(3)$ & & \\
\hline Cell communication & 0.436129 & 20 (19) & MAPK1, GRB2 & PTPN11 \\
\hline Cell cycle & 0.537926 & $15(6)$ & MAPK1 & \\
\hline Lipid metabolic process & 0.931277 & $7(5)$ & & \\
\hline $\begin{array}{l}\text { Nucleobase, nucleoside, nucleotide } \\
\text { and nucleic acid metabolic process }\end{array}$ & 0.946888 & $38(30)$ & RUVBL2, MAPK1 & \\
\hline \multicolumn{5}{|l|}{ Cellular component } \\
\hline Cytosol & $7.25 \mathrm{E}-11^{\mathrm{a}}$ & $45(26)$ & MAPK1, GRB2 & PTPN11 \\
\hline Mitochondrion & $5.85 \mathrm{E}-07^{\mathrm{a}}$ & $20(26)$ & MAPK1 & \\
\hline Endosome & $0.006439^{\mathrm{a}}$ & $6(9)$ & GRB2 & \\
\hline Golgi apparatus & $0.009079^{\mathrm{a}}$ & $10(16)$ & GRB2 & \\
\hline Endoplasmic reticulum & 0.012008 & $13(14)$ & & \\
\hline Extracellular region & 0.013983 & $28(18)$ & & \\
\hline Cytoskeleton & 0.024579 & $18(18)$ & TMSB10, MAPK1 & \\
\hline Lysosome & 0.026232 & $4(5)$ & & \\
\hline Vacuole & 0.031176 & $5(5)$ & & \\
\hline Peroxisome & 0.493033 & $1(1)$ & & \\
\hline Ribosome & 0.628763 & $2(1)$ & & \\
\hline Plasma membrane & 0.844492 & $27(31)$ & & \\
\hline Nucleus & 0.963673 & $41(33)$ & RUVBL2, MAPK1, GRB2 & \\
\hline \multicolumn{5}{|l|}{ Molecular function } \\
\hline Protein binding & $2.61 \mathrm{E}-06^{\mathrm{a}}$ & $73(64)$ & $\begin{array}{l}\text { TMSB10, RUVBL2, PTPN11 } \\
\text { MAPK1, GRB2 }\end{array}$ & \\
\hline Structural molecule activity & $0.000156^{\mathrm{a}}$ & $11(12)$ & & \\
\hline Oxygen binding & $0.00194^{\mathrm{a}}$ & $3(1)$ & & \\
\hline Catalytic activity & 0.070336 & 47 (49) & RUVBL2, MAPK1 & PTPN11 \\
\hline Nucleotide binding & 0.09617 & $28(18)$ & RUVBL2, MAPK1 & \\
\hline Carbohydrate binding & 0.099071 & $7(3)$ & & \\
\hline Lipid binding & 0.126594 & $10(1)$ & & \\
\hline Enzyme regulator activity & 0.163896 & $13(6)$ & & \\
\hline Transporter activity & 0.54845 & $12(6)$ & & \\
\hline Motor activity & 0.587484 & $1(1)$ & & \\
\hline Signal transducer activity & 0.900132 & $14(13)$ & MAPK1, GRB2 & \\
\hline $\begin{array}{l}\text { Transcription regulator } \\
\text { activity }\end{array}$ & 0.939697 & $2(2)$ & & \\
\hline Nucleic acid binding & 0.990735 & $18(20)$ & RUVBL2, MAPK1 & \\
\hline
\end{tabular}

${ }^{\text {aP }}<0.05$ was considered a statistically significant difference. Genes listed had high interaction counts with other genes and were considered of higher significance. 
Table V. Kyoto Encyclopedia of Genes and Genomes pathways of the differentially expressed proteins in Down syndrome.

\begin{tabular}{|c|c|c|c|}
\hline Pathway & P-value ${ }^{a}$ & $\begin{array}{l}\text { Upregulated } \\
\text { (downregulated) } \\
\text { gene number }\end{array}$ & $\begin{array}{c}\text { Significantly } \\
\text { upregulated genes }\end{array}$ \\
\hline Complement and coagulation cascades & 1.43E-09 & $14(2)$ & PLG \\
\hline Focal adhesion & 0.001385 & $7(10)$ & PTK2, MAPK1, FN1, GRB2 \\
\hline Chagas disease (American trypanosomiasis) & 0.003458 & $6(4)$ & MAPK1 \\
\hline Pertussis & 0.010577 & $7(0)$ & MAPK1 \\
\hline Pyruvate metabolism & 0.025336 & $2(2)$ & \\
\hline Proteasome & $1.06 \mathrm{E}-06$ & $6(4)$ & \\
\hline Oxidative phosphorylation & 0.044748 & $5(4)$ & \\
\hline Fc gamma R-mediated phagocytosis & 0.005818 & $2(7)$ & MAPK1 \\
\hline Amoebiasis & 0.033113 & $4(4)$ & PTK2, FN1 \\
\hline Glycolysis/gluconeogenesis & 0.016705 & $4(2)$ & \\
\hline Thyroid cancer & 0.029584 & $1(2)$ & MAPK1 \\
\hline Pathogenic Escherichia coli infection & 0.006884 & $3(3)$ & \\
\hline Glyoxylate and dicarboxylate metabolism & 0.035541 & $1(1)$ & \\
\hline Staphylococcus aureus infection & $2.46 \mathrm{E}-06$ & $10(1)$ & PLG \\
\hline Systemic lupus erythematosus & 0.026282 & $6(4)$ & \\
\hline Prion diseases & 0.002715 & $5(0)$ & MAPK1 \\
\hline Folate biosynthesis & 0.014487 & $0(2)$ & \\
\hline Ribosome & 0.032953 & $3(4)$ & \\
\hline Alanine, aspartate and glutamate metabolism & 0.040724 & $2(1)$ & \\
\hline ECM-receptor interaction & 0.000658 & $2(8)$ & FN1 \\
\hline Cell adhesion molecules & 0.04671 & $3(6)$ & \\
\hline Shigellosis & 0.01202 & $1(5)$ & MAPK1 \\
\hline Amino sugar and nucleotide sugar metabolism & 0.000664 & $3(4)$ & \\
\hline Citrate cycle (TCA cycle) & 0.00118 & $4(1)$ & \\
\hline RNA transport & 0.000224 & $6(10)$ & \\
\hline Galactose metabolism & 0.029584 & $2(1)$ & \\
\hline Bacterial invasion of epithelial cells & 0.000115 & $3(7)$ & PTK2, FN1 \\
\hline African trypanosomiasis & 0.00363 & $5(0)$ & \\
\hline
\end{tabular}

${ }^{a} \mathrm{P}<0.05$ was considered to indicate a statistically significant difference. Genes listed had high interaction counts with other genes and were considered of higher significance.

having an important regulatory role in cell biology (23). miR-27b and miR-27a have been found to negatively regulate adipocyte differentiation through the post-transctiptional regulation of the peroxisome proliferator-activated receptor $\gamma(24)$. These findings suggest that miRNAs with significantly differential levels of expression are key in cell differentiation in DS. The focus of the present study was not centred on comparing miRNA between normal and DS samples. Therefore, more comprehensive clinical investigations are required to characterize the differential expression of the miRNA identified in DS.

As iTRAQ has previously been suggested to be suitable for identifying novel plasma biomarkers (25), this method has been used to detect for potential quantitative changes in the plasma proteome of fetuses with DS, compared with normal fetuses. These proteins are found in the sera of patients with Alzheimer's disease, which has a similar pathology to DS (26). In addition to the miRNA profile, the present study described changes of the protein expression profile using iTRAQ. As a result, several proteomic changes in DS were revealed. A number of genes were identified in the analyses, and a comprehensive analysis of protein complexes, which may be coordinately regulated by miRNAs was performed. Gene network methods provide novel insights for elucidating the complexity of diseases, including DS. Hub nodes have been found to be key in several networks. Hub genes with high levels of connection are expected to be important in biology (27). In the present study, GRB2, TMSB10, RUVBL2, MAPK1, PTPN11, PTK2 and ACTA2 were identified as hub genes. These may be important in biological process, cellular component and molecular function in DS, and the proteins identified in DS each require in depth examination in order to understand their functional relevance.

Inhibition of the function of GRB2 hinders the proliferation and transformation of various cell types and impairs developmental processes in various organisms. Thus, it is not unexpected that a targeted gene disruption of GRB2 is lethal at 


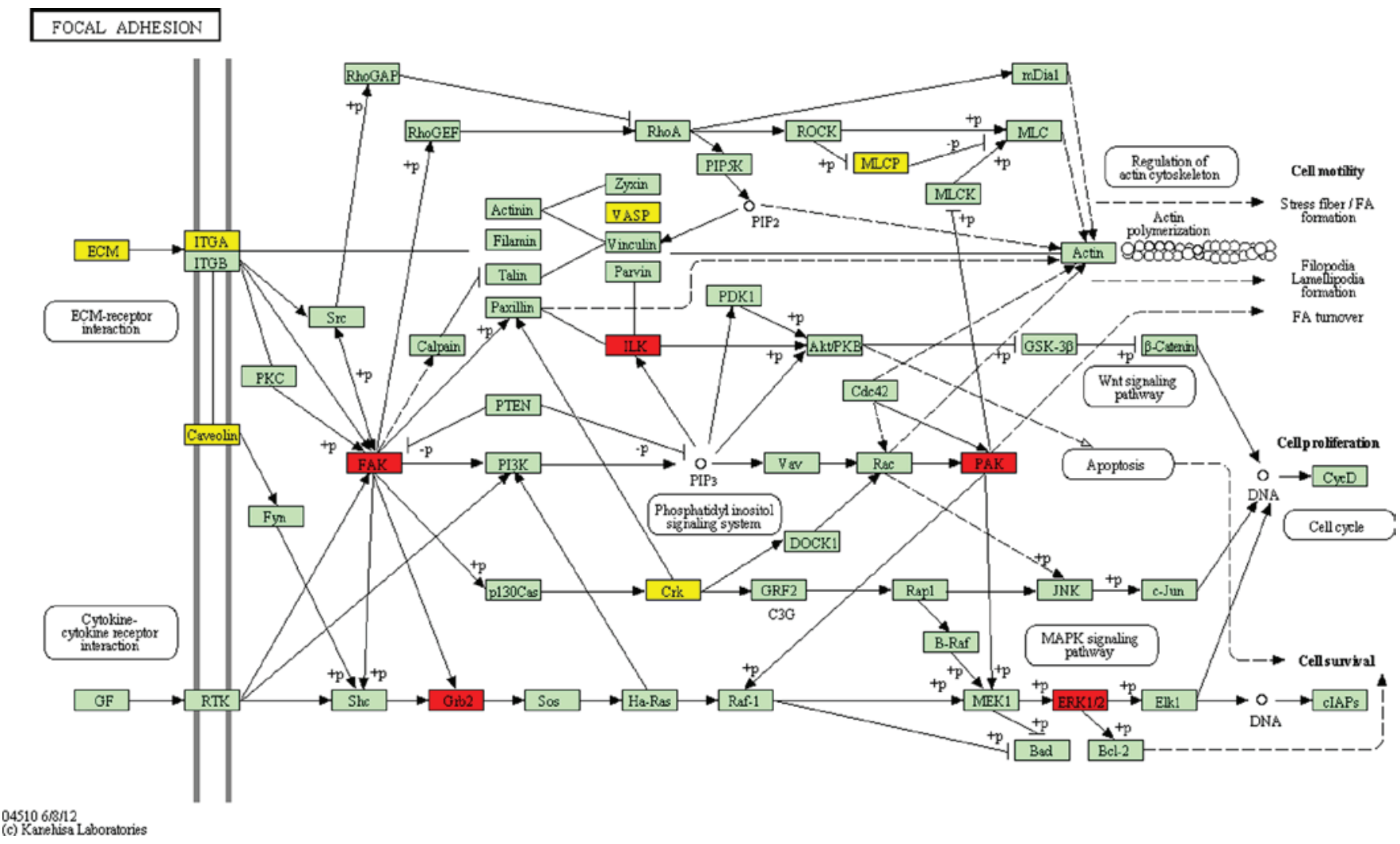

Figure 3. Focal adhesion pathway of the differentially expressed proteins in Down syndrome. Red indicates an upregulated gene; yellow indicates a downregulated gene.

an early embryonic stage. Nonchimeric polytransgenic 152F7 mice, which have four human chromosome 21 genes within the DS critical region, present with learning and memory impairment. Decreased levels of GRB2 in the $152 \mathrm{~F} 7$ mice may contribute to impaired cytoskeletak functions in the hippocampus (28). $R U V B L 2$ is important in DNA damage repair, transcriptional regulation and chromatin remodeling (29). PTK2 is a focal adhesion-associated protein kinase involved in spreading processes and cellular adhesion. Noonan syndrome is a fairly common autosomal, dominantly-inherited disorder. It is the most common syndromal cause of congenital heart disease following DS. In the case of Noonan syndrome, genetic diseases associated with PTPN11, mutations are broadly distributed in the coding region of the gene, however, all appear to lead to unregulated, or hyperactivated mutant forms of the protein (30). Impaired signaling in DS involving different signaling systems has been suggested. In addition, the availability of fetal brain and proteome technologies, identifying individual brain proteins, including $M A P K 1$, led to the present study investigating individual signaling factors in the brain (31). The functional analysis of the miRNA-regulated protein complexes showed a clear bias towards signal transduction, transcriptional regulation, chromatin regulation and cell cycle. The method used in the present study provided improved candidate miRNA target lists, as demonstrated by a benchmark against large-scale, quantitative proteomics data.

The present study identified more than one potential miRNA-target pair from the predicted targets. Functional annotation indicated that they were involved in clusters of meaningful and significantly relevant biological processes.
Using the target analysis method enabled identification of the miRNA targets affected at the protein level. In order to elucidate the functions of the targets of miRNAs, KEGG pathway and GO term annotation were used to their target gene pool. KEGG annotation showed a significant change in the focal adhesions pathway in the DS group, compared with the normal group. Further investigation of the miRNA-gene network of the pathway showed that hsa-miR-329 may be the key regulators of the focal adhesion pathway. Focal adhesion showed a high level of enrichment and representation in the present study. This pathway includes several proteins, including $M A P K$. MAPK pathways can regulate cellular functions, including differentiation, proliferation, apoptosis and migration (32). Therefore, although the exact mechanism remains to be fully elucidated and requires further investigation, miRNAs may be involved in DS by regulating cell proliferation, differentiation and the cell signaling network. Their regulatory roles in the focal adhesions pathway may be involved in the pathogenesis of the DS.

In the present study, the miRNA target prediction and large-scale protein-protein interaction data used was found to be useful for improving current biological knowledge. Taken together, the three miRNAs (hsa-miR-329, hsa-miR-27b and hsa-miR-27a) and seven proteins (GRB2, TMSB10, RUVBL2, MAPK1, PTPN11, ACTA2 and PTK2) with the highest level of differential expression in the DS fetuses were identified. The results also identified several directions for future investigations. Each possible miRNA-protein pair, which was identified in the present study, is a candidate for further extensive investigation to definitively confirm the presence of specific miRNA-protein interactions, thus providing a more 
detailed understanding of the pathogenesis of DS. miRNAs and their target genes maintain a balance of gene expression regulatory networks; if this balance is disrupted, it leads to disease. Therefore, changes in specific miRNA and protein levels may affect gene expression in DS. An understanding of the gene regulatory networks controlled by miRNA in conjunction with protein in DS is required. The present study indicated that miRNAs are probable factors and potential biomarkers involved in the pathogenesis of DS. Further investigations are required to understand the roles of the identified miRNAs in the pathogenesis of DS. Integrating miRNA and protein data sets is a promising strategy for understanding the pathogenesis of DS. The findings of the present study provided insight into the potential contribution of anomalous regulated miRNAs to the abnormalities in DS. This may assist in structuring antenatal diagnostic biomarkers of DS, and identify novel therapeutic targets for the treatment of individuals with DS. The investigation of miRNAs may also lead to the identification of novel methods to prevent and treat other diseases.

\section{Acknowledgements}

This study was financially supported by the Shenzhen Municipal Science and Technology Innovation Council, People's Republic of China (grant no. 201202121 and key project CXZZ20130321090846345) and the Guangdong Provincial S\&T Program (grant no. 2012B032000008).

\section{References}

1. Xu Y, Li W, Liu X, Ma H, Tu Z and Dai Y: Analysis of microRNA expression profile by small RNA sequencing in down syndrome fetuses. Int J Mol Med 32: 1115-1125, 2013.

2. Pellegrini FP, Marinoni M, Frangione V, Tedeschi A, Gandini V, Ciglia F, Mortara L, Accolla RS and Nespoli L: Down syndrome, autoimmunity and T regulatory cells. Clin Exp Immunol 169: 238-243, 2012 .

3. Jin S, Lee YK, Lim YC, Zheng Z, Lin XM, Ng DP, Holbrook JD, Law HY, Kwek KY, Yeo GS and Ding C. Global DNA hypermethylation in down syndrome placenta. PLoS Genet 9: e1003515, 2013.

4. Costa V, Angelini C, D'Apice L, Mutarelli M, Casamassimi A, Sommese L, Gallo MA, Aprile M, Esposito R, Leone L, et al: Massive-scale RNA-Seq analysis of non ribosomal transcriptome in human trisomy 21. PLoS One 6: e18493, 2011.

5. Wang J, Haubrock M, Cao KM, Hua X, Zhang CY, Wingender E and Li J: Regulatory coordination of clustered microRNAs based on microRNA-transcription factor regulatory network. BMC Syst Biol 5: 199, 2011.

6. Hwang HW and Mendell JT: MicroRNAs in cell proliferation, cell death, and tumorigenesis. Br J Cancer 96 (Suppl): R40-R44, 2007.

7. Liao JM, Zhou X, Zhang Y and Lu H: MiR-1246: A new link of the p53 family with cancer and down syndrome. Cell Cycle 11: 2624-2630, 2012.

8. Elton TS, Sansom SE and Martin MM: Trisomy-21 gene dosage over-expression of miRNAs results in the haploinsuffciency of specifc target proteins. RNA Biol 7: 540-547, 2010.

9. Malinge S, Izraeli S and Crispino JD: Insights into the manifestations, outcomes, and mechanisms of leukemogenesis in down syndrome. Blood 113: 2619-2628, 2009.

10. Ozen M, Creighton CJ, Ozdemir M and Ittmann M: Widespread deregulation of microRNA expression in human prostate cancer. Oncogene 27: 1788-1793, 2008.

11. Elkan-Miller T, Ulitsky I, Hertzano R, Rudnicki A, Dror AA, Lenz DR, Elkon R, Irmler M, Beckers J, Shamir R and Avraham KB: Integration of transcriptomics, proteomics, and MicroRNA analyses reveals novel MicroRNA regulation of targets in the mammalian inner ear. PLoS One 6: e18195, 2011.
12. Vasilatou D, Papageorgiou S, Pappa V, Papageorgiou E and Dervenoulas J: The role of microRNAs in normal and malignant hematopoiesis. Eur J Haematol 84: 1-16, 2010.

13. Li R, Yu C, Li Y, Lam TW, Yiu SM, Kristiansen K and Wang J: SOAP2: An improved ultrafast tool for short read alignment. Bioinformatics 25: 1966-1967, 2009.

14. Livak KJ and Schmittgen TD: Analysis of relative gene expression data using real-time quantitative PCR and the 2 (-Delta Delta C (T)) Method. Methods 25: 402-408, 2001

15. Wang L, Dai Y, Qi S, Sun B, Wen J, Zhang L and Tu Z: Comparative proteome analysis of peripheral blood mononuclear cells in systemic lupus erythematosus with iTRAQ quantitative proteomics. Rheumatol Int 32: 585-593, 2012.

16. Sui W, Lin H, Peng W, Huang Y, Chen J, Zhang Y and Dai Y: Molecular dysfunctions in acute rejection after renal transplantation revealed by integrated analysis of transcription factor, microRNA and long noncoding RNA. Genomics 102: 310-322, 2013.

17. Gao W, Xu J, Liu L, Shen H, Zeng $H$ and Shu Y: A systematic-analysis of predicted miR-21 targets identifies a signature for lung cancer. Biomed Pharmacother 66: 21-28, 2012.

18. Flynt AS and LaiEC: Biological principles of microRNA-mediated regulation: Shared themes amid diversity. Nat Rev Genet 9: 831-842, 2008.

19. Corsini LR, Bronte G, Terrasi M, Amodeo V, Fanale D, Fiorentino E, Cicero G, Bazan V and Russo A: The role of microRNAs in cancer: Diagnostic and prognostic biomarkers and targets of therapies. Expert Opin Ther Targets 16 (Suppl 2): S103-S109, 2012.

20. Keck-Wherley J, Grover D, Bhattacharyya S, Xu X, Holman D, Lombardini ED, Verma R, Biswas R and Galdzicki Z: Abnormal microRNA expression in Ts65Dn hippocampus and whole blood: Contributions to down syndrome phenotypes. Dev Neurosci 33: 451-467, 2011.

21. Elton TS, Sansom SE and Martin MM. Trisomy-21 gene dosage overexpression of miRNAs results in the haploinsufficiency of specific target proteins. RNA Biol 7: 540-547, 2010.

22. Xiao B, Tan L, He B, Liu Z and Xu R: MiRNA-329 targeting E2F1 inhibits cell proliferation in glioma cells. J Transl Med 11: 172,2013

23. Wang Y, Rathinam R, Walch A and Alahari SK: ST14 (suppression of tumorigenicity 14) gene is a target for miR-27b, and the inhibitory effect of ST14 on cell growth is independent of miR-27b regulation. J Biol Chem 284: 23094-23106, 2009.

24. Kim SY, Kim AY, Lee HW, Son YH, Lee GY, Lee JW, Lee YS and Kim JB: miR-27a is a negative regulator of adipocyte differentiation via suppressing PPARgamma expression. Biochem Biophys Res Commun 392: 323-328, 2010.

25. Sui W, Zhang R, Chen J, He H, Cui Z, Ou M, Li W, Qi S, Wen J, Lin X and Dai Y: Quantitative proteomic analysis of Down syndrome in the umbilical cord blood using iTRAQ. Mol Med Rep 11: 1391-1399, 2015.

26. Kolla V, Jenö P, Moes S, Tercanli S, Lapaire O, Choolani M and Hahn S: Quantitative proteomics analysis of maternal plasma in down syndrome pregnancies using isobaric tagging reagent (iTRAQ). J Biomed Biotechnol 2010: 952047, 2010.

27. Langfelder P, Mischel PS and Horvath S: When is hub gene selection better than standard meta-analysis. PLoS One 8: e61505, 2013

28. Shin JH, Guedj F, Delabar JM and Lubec G: Dysregulation of growth factor receptor-bound protein 2 and fascin in hippocampus of mice polytransgenic for chromosome 21 structures. Hippocampus 17: 1180-1192, 2007.

29. Gorynia S, Bandeiras TM, Pinho FG, McVey CE, Vonrhein C, Round A, Svergun DI, Donner P, Matias PM and Carrondo MA: Structural and functional insights into a dodecameric molecular machine-the RuvBL1/RuvBL2 complex. J Struct Biol 176: 279-291, 2011.

30. Essawi ML, Ismail MF, Afifi HH, Kobesiy MM, El Kotoury A and Barakat MM: Mutational analysis of the PTPN11 gene in Egyptian patients with Noonan syndrome. J Formos Med Assoc 112: 707-712, 2013.

31. Peyrl A, Weitzdoerfer R, Gulesserian T, Fountoulakis M and Lubec G: Aberrant expression of signaling-related proteins 14-3-3 gamma and RACK1 in fetal down syndrome brain (trisomy 21). Electrophoresis 23: 152-157, 2002.

32. Slattery ML, Lundgreen A and Wolff RK: Dietary influence on MAPK-signaling pathways and risk of colon and rectal cancer. Nutr Cancer 65: 729-738, 2013. 